\title{
TNFAIP8 Regulates Intestinal Epithelial Cell Differentiation and May Alter Terminal Differentiation of Secretory Progenitors
}

\author{
Ryan Hood, Youhai H. Chen and Jason R. Goldsmith *,+(D)
}

check for

updates

Citation: Hood, R.; Chen, Y.H.; Goldsmith, J.R. TNFAIP8 Regulates Intestinal Epithelial Cell

Differentiation and May Alter Terminal Differentiation of Secretory Progenitors. Cells 2021, 10, 871.

https://doi.org/10.3390/cells10040871

Academic Editor: Alexander

V. Ljubimov

Received: 5 March 2021

Accepted: 8 April 2021

Published: 12 April 2021

Publisher's Note: MDPI stays neutral with regard to jurisdictional claims in published maps and institutional affiliations.

Copyright: (c) 2021 by the authors. Licensee MDPI, Basel, Switzerland. This article is an open access article distributed under the terms and conditions of the Creative Commons Attribution (CC BY) license (https:// creativecommons.org/licenses/by/ $4.0 /)$.
Department of Pathology and Laboratory Medicine, Perelman School of Medicine, 422 Curie Blvd, Philadelphia, PA 19104, USA; ryanhood@sas.upenn.edu (R.H.); yhc@pennmedicine.upenn.edu (Y.H.C.)

* Correspondence: goldsj@pennmedicine.upenn.edu

+ Currently Employed as Director of Donor Medical Sciences, Seres Therapeutics, Inc., 200 Sidney Street, Cambridge, MA 02139, USA.

\begin{abstract}
The intestine is a highly proliferative dynamic environment that relies on constant selfrenewal of the intestinal epithelium to maintain homeostasis. Tumor necrosis factor-alpha-induced protein 8 (TNFAIP8 or TIPE0) is a regulator of PI3K-mediated signaling. By binding to PIP2 and PIP3, TIPE family members locally activate PI3K activity while globally inhibiting PI3K activity through sequestration of membranous PIP2. Single-cell RNA sequencing survey of Tipe $0^{-/-}$small intestine was used to investigate the role of TIPE0 in intestinal differentiation. Tipe $0^{-/-}$intestinal cells were shown to shift towards an undifferentiated state, with the notable exception of goblet cells. Additionally, three possible novel regulators of terminal cell fate decisions in the secretory lineage were identified: Nupr1, Kdm4a, and Gatad1. We propose that these novel regulators drive changes involved in goblet cell (Nupr1) or tuft cell (Kdm4a and Gatad1) fate commitment and that TIPE0 may play a role in orchestrating terminal differentiation.
\end{abstract}

Keywords: cell fate decisions; secretory progenitors; pseudotime; homeostasis; transcriptional regulation

\section{Introduction}

Intestinal homeostasis relies on rapid and continuous self-renewal of the intestinal epithelium controlled by various inputs and signaling pathways. Intestinal stemness is regulated by the $\mathrm{Wnt} / \beta$-catenin pathway, which maintains intestinal stem cell (ISC) selfrenewal by mediating expression of ISC-specific genes [1]. The Wnt/ $\beta$-catenin pathway is also involved in determining the secretory lineage in the absence of Notch signaling [2]. Additionally, mouse atonal homologue 1 (Math1 or Atoh1) is required for entry into the secretory lineage [3-5]. Activated Notch signaling, on the other hand, determines commitment to the absorptive lineage by upregulation of Hes1, an inhibitor of Atoh1. Terminal differentiation in the secretory lineage involves a number of different factors, including neurogenin 3 (Neurog3) in enteroendocrine cells [6-8], Kruppel-like factor 4 (Klf4) and sterile alpha motif pointed domain containing ETS transcription factor (Spdef) in goblet cells [9-11], growth factor independent 1 (Gfi1) in Paneth cells [12], and Gfi1b in tuft cells [13]. The balance between proliferation and differentiation is further mediated by a number of additional pathways, including Hedgehog, bone morphogenic protein (BMP), PI3K/Akt, Jak/STAT, and Eph-ephrin signaling pathways [14].

Tumor necrosis factor-alpha-induced protein 8 (TNFAIP8 or TIPE0) is a member of the TNFAIP8-like (TIPE) family of proteins along with TNFAIP8L1-3 (TIPE1-3) [15-17]. TIPE0, as well as other members of the TIPE family, has been found to interact with a variety of phospholipids, including phosphatidylinositol-4-phosphate (PI4P), PI (4,5) P2, PI $(3,5)$ P2, PI $(3,4)$ P2, and PI $(3,4,5)$ P3 through a large hydrophobic TIPE homology $(\mathrm{TH})$ domain [18-20]. Through binding to PIPs, TIPE0 plays an important role in modulating 
PI3K signaling. ADP-Glo PI3K assay demonstrated dose-dependent ATP consumption and potentiation of PI $(3,4,5)$ P3 with addition of increasing concentrations of TIPE0, which suggests TIPE0's ability to locally activate PI3K signaling [21]. Recently, however, mass ELISA in ileal and colonic samples and co-localization analysis using a PI $(3,4,5)$ P3 biosensor demonstrated increased PI $(3,4,5)$ P3 in the Tipe $0^{-/-}$intestine and in the cell membrane of Tipe $0^{-/-}$cells [22]. Global increase in PI (3-5) P3 also elevated PI3K/Akt signaling in Tipe $0^{-/-}$intestinal epithelium, suggesting that TIPE0 may globally suppress PI3K signaling [22]. Furthermore, aberrant PI3K/Akt signaling in the Tipe $0^{-/-}$intestine was found to alter the cellular composition of the intestinal epithelium, causing a shift towards an undifferentiated state [22]. In this study, we use this phenomenon of altered differentiation in the Tipe $0^{-/-}$gut, coupled to single-cell RNA-Seq pseudotime analyses, to discover novel regulators of secretory lineage fate commitment.

\section{Materials and Methods}

\subsection{Animals}

Wild-type (WT) C57BL/ 6 mice were purchased from the Jackson Laboratory (Bar Harbor, ME, USA) or bred in house. Tipe $0^{-/-}$mice were previously generated, as described elsewhere [23]. WT and Tipe $0^{-1-}$ mice used in the study were colony controlled (from the same initial litter) but were propagated separately. All mice used in this study were housed under pathogen-free conditions in the University of Pennsylvania Animal Care Facilities. All animal protocols used were pre-approved by the Institutional Animal Care and Use Committee of the University of Pennsylvania.

\subsection{Single Cell RNA Sequencing Analysis}

scRNA-seq dataset was generated by Goldsmith et al. [22]. All functions used in this study are either part of R base-functions or of the packages Monocle 3 [24-26], Dplyr, Matrix, or Tidyverse.

\subsubsection{Dimensionality Reduction Using PCA and UMAP}

Pre-processing of droplet (10X) scRNA-seq data including demultiplexing, alignment to the mm10 transcriptome, and UMI-collapsing was performed by Goldsmith et al. [22]. 10X genomics output was loaded into $R$ as a cell data set (CDS) object using the "load_mm_data" function from the Monocle3 package. A metadata column containing the short names of each gene was specified in addition to the 10X genomics output. The processed data was normalized by logarithmic and size factors to address differences in sequencing depth followed by dimensionality reduction by Principal Component Analysis (PCA) using the Monocle3 function "preprocess_cds". Fourteen principal components were used as described in Goldsmith et al. [22]. The dimensionality of the data was further reduced for visualization by using Uniform Manifold Approximation and Projection (UMAP). This was implemented using the default parameters of the "reduce_dimension" function from the Monocle3 package.

\subsubsection{Clustering and Annotation}

Cells were grouped into clusters using Leiden-based community detection as reported by Levine et al. using the Monocle function "cluster_cells" [27]. This was performed with a k-parameter of 40 and a resolution of $1 \times 10^{-2}(\mathrm{WT})$ or $4 \times 10^{-2}\left(\mathrm{TKO}\right.$, i.e., Tipe $0^{-/-}$). Marker genes by cluster were determined using the Monocle function "top_markers" with the default number of cores and reference cells. Gene markers from Haber et al. were used to identify the cell types of each cluster [27]. Supplementary data from Haber et al. was reformatted to fit into a comma-delimited format and loaded into R. Then, a Monocle-3specific function, "clust_marker_topx", was written to generate a data frame that classifies top markers for each cluster by cell type according to Haber et al. First, the output of "top_markers" was filtered to exclude markers for which the fraction expressing was less than 0.10. Next, the gene name for all identified markers for a given cluster was stored as 
a vector. This vector was then iteratively compared to the cell type markers from Haber et al., and similar markers were stored in a data frame whose columns corresponded to the associated cell type. Using this function, the cell type identities of each cluster were determined. Cells were then annotated by storing a character vector as a column of "colData" of the CDS object. Initial annotation was sufficient for the WT CDS. However, the TKO UMAP plot lacked clusters with markers of IELs and enteroendocrine cells. These cell types were identified by plotting IEL or enteroendocrine marker gene expression on the UMAP plot. These cells were then visually subsetted using "choose_cells" and annotated as described above. Furthermore, substructure in clusters 3 and 10 was identified by visually subsetting the individual clusters as described above and clustering these cells separately. Further annotations were then added to the CDS as above.

\subsubsection{Determining Cell Type Percentages}

A Monocle-3-specific-function, "cell_type_percentages", was written to determine the number of cells in each subset by assigned cell type. First, the length of the vector "rownames" of "colData", which contains the cell barcodes, was determined representing the total number of cells in the CDS. Next, this was done for the "colData" matrix subsetted by cell type representing the total number of cells of each type. The number of cells of each subtype was then divided by the total number of cells and multiplied by 100 , yielding the percentage of the total composed of each cell type.

\subsubsection{Learning Trajectories and Ordering Cells in Pseudotime}

Trajectories were learned by Monocle $3^{\prime}$ s reversed graph embedding algorithm using the function "learn_graph" [26]. Subsequently, cells were ordered in pseudotime using the function "order_cells" with a selected node representing Lgr5+ stem cells to define the 0 point of pseudotime.

\subsubsection{Pseudotime Binning Analysis}

A Monocle-3-specific function was written to subset cells by pseudotime, fit a regression to each by genotype, and generate a data frame with the significant differences in expression for identified genes by pseudotime bin. This was done by storing each cell's pseudotime value to the "colData" matrix. The CDSs of each genotype were combined using the function "combine_cds", which automatically adds sample (in this case genotype) to the "colData" of the combined CDS. The combined CDS was input to the function "pseudotime_bin_analysis". First, the combined CDS was subsetted by single-unit pseudotime bins and a regression model was fit to each gene to compare expression between genotypes. The output was then filtered to remove intercept values and non-significant genes. Genes differentially expressed across different pseudotime bins were stored as a vector, excluding repeating values. A data frame was then generated, with the first column containing the short names of identified genes and the following columns containing NA values. These NA values were then replaced with the significant regression values for each gene. The function then returned a list of the generated data frame as well as the individual outputs for each pseudotime bin.

\subsubsection{Secretory Branch Analysis}

The secretory branch was identified by plotting the secretory precursor Atoh1 on the UMAP plot for each genotype. The branch was isolated using the "choose_cells" function from Monocle 3 and manually selecting the cells along the trajectories identified above. Regression analysis was performed on each genotype separately using genotype as the independent variable. The regression for each genotype was then compared by student's $t$-test. Gene expression was plotted across pseudotime using the function "plot_genes_in_pseudotime". 


\section{Results}

\subsection{Loss of TIPE0 Alters Intestinal Differentiation}

Single-cell RNA sequencing (scRNA-seq) was used to confirm the effect of TIPE0 knockout on the composition of the ileal epithelium. Monocle 3 was used to cluster cells at a resolution of $1 \times 10^{-2}$ for WT $(n=3402)$ and $4 \times 10^{-2}$ for Tipe $^{-1-}(n=2006)$. Larger resolution values result in more clusters [28], which was necessary for proper determination of cell types in the smaller dataset. Seventeen clusters were identified for each genotype, and two additional clusters were identified in the Tipe $0^{-/-}$dataset (representing intraepithelial lymphocytes (IELs) and enteroendocrine cells (EECs)). Additionally, substructure was identified for clusters 3 and 10 representing tuft cells and another cluster of goblet cells, respectively. Clusters were then annotated using established gene profiles for the small intestinal epithelium. Clusters representing each of the cell types found in the ileal epithelium were identified as well as a cluster in each sample representing intraepithelial lymphocytes (Figure 1a). Tipe $0^{-/-}$mice had similar or decreased levels of terminally differentiated enterocytes and increased levels of transitional/partially differentiated and Lgr5+ stem cells, consistent with previous studies (Figure 1b) [22]. Goblet cells were significantly enriched in the Tipe $0^{-/-}$intestine, which is also consistent with previous findings by Goldsmith et al. These data further suggest that differentiation in the Tipe $0^{-/-}$intestine is dysregulated, with a shift from terminally differentiated enterocytes towards Lgr5+ stem cells and transitional enterocytes with the notable exception of goblet cells.

\section{a}
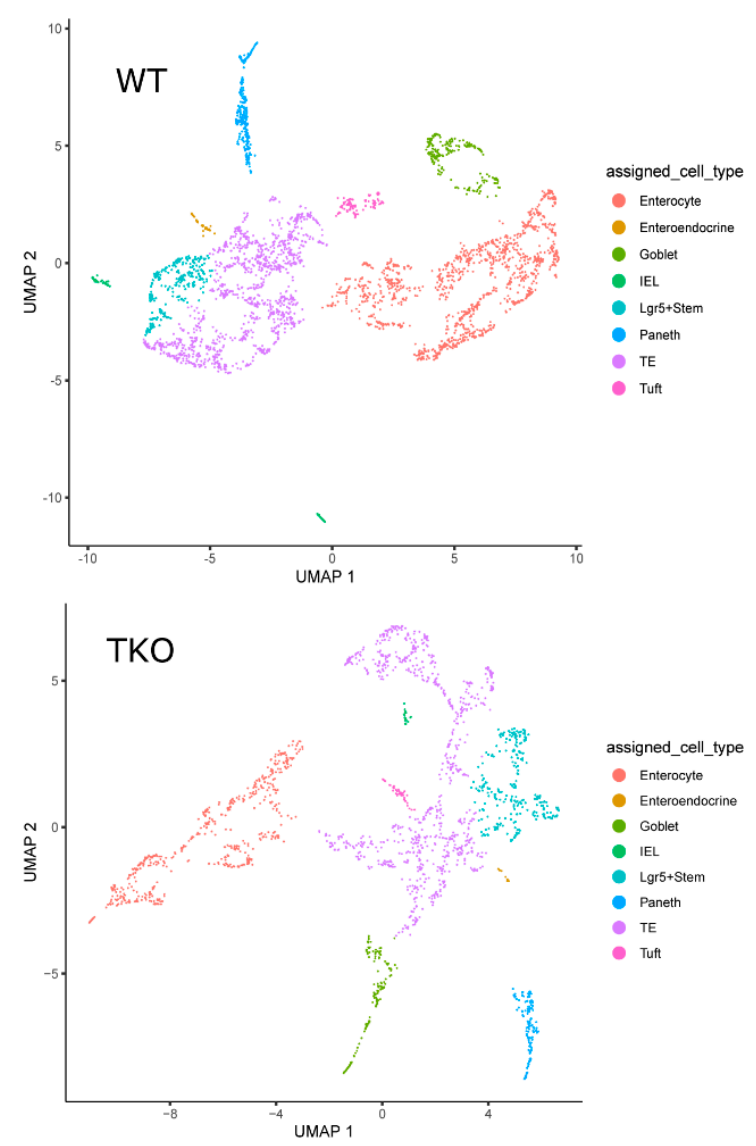

b

\begin{tabular}{|l|c|c|c|}
\hline & WT (\%) & TKO (\%) & $p$-value \\
\hline Enterocyte & 34.71 & 25.32 & $<0.00001$ \\
\hline TE & 34.57 & 40.93 & $<0.00001$ \\
\hline Lgr5+Stem & 8.642 & 13.36 & $<0.00001$ \\
\hline Paneth & 8.172 & 6.879 & $<0.05$ \\
\hline Goblet & 6.878 & 11.86 & $<0.00001$ \\
\hline Tuft & 3.233 & 2.094 & $<0.05$ \\
\hline EEC & 1.205 & 0.8475 & n.s. \\
\hline IEL & 2.587 & 1.196 & $<0.0005$ \\
\hline
\end{tabular}

Figure 1. TKO (Tipe $0^{-/-}$) intestine is shifted towards an undifferentiated state. (a) Uniform Manifold Approximation and Projection (UMAP) plots colored by cell type, $n=3$ mice/genotype, wild-type (WT): 3402 cells, TKO: 2006 cells; (b) Tabulated percentages of cell types, colored by difference between TKO and WT (purple (negative), yellow (positive)). Significance determined by $z$-test for independent proportions. n.s. = not significant. 


\subsection{Loss of TIPE Leads to Altered Gene Expression in the Intestinal Epithelium}

Given that TIPE0 modulates PI3K/Akt signaling and that expression of stem cell markers and beta-catenin targets are altered by loss of TIPE0, we investigated which genes may be responsible for the changes in the composition of the Tipe $0^{-/-}$intestine. Monocle 3 was employed to construct single-cell trajectories that reflect the kinetics of various gene modules (Figure 2a). Based on these trajectories, cells were then ordered in pseudotime, defining the node at Lgr5+ stem cells (Figure 2b). Due to technical limitations, regression analysis using both genotype and pseudotime as continuous independent variables could not be performed. Thus, pseudotime was grouped into one-unit bins and regression using genotype was performed for each bin. This analysis revealed 298 differentially expressed genes between WT and Tipe $0^{-/-}$through different points in pseudotime. Thirty genes were then selected for further analysis based on capacity to regulate transcription or modulate signaling pathways involved in intestinal epithelial cell differentiation (Figure 2d). However, for the identified genes, differential expression was restricted to early time points with no significant difference at later time points. This was likely due to the varying trajectories that composed each point in pseudotime following differentiation of Lgr5+ stem cells into secretory and absorptive precursors that would affect the measure level of expression at a given time point. Thus, using the genes identified by this analysis, it was necessary to select a single major branch for further analysis (Figure 2c). Despite this, these data further suggest that TIPE0 is involved in modulating expression programs that regulate differentiation in the intestine.
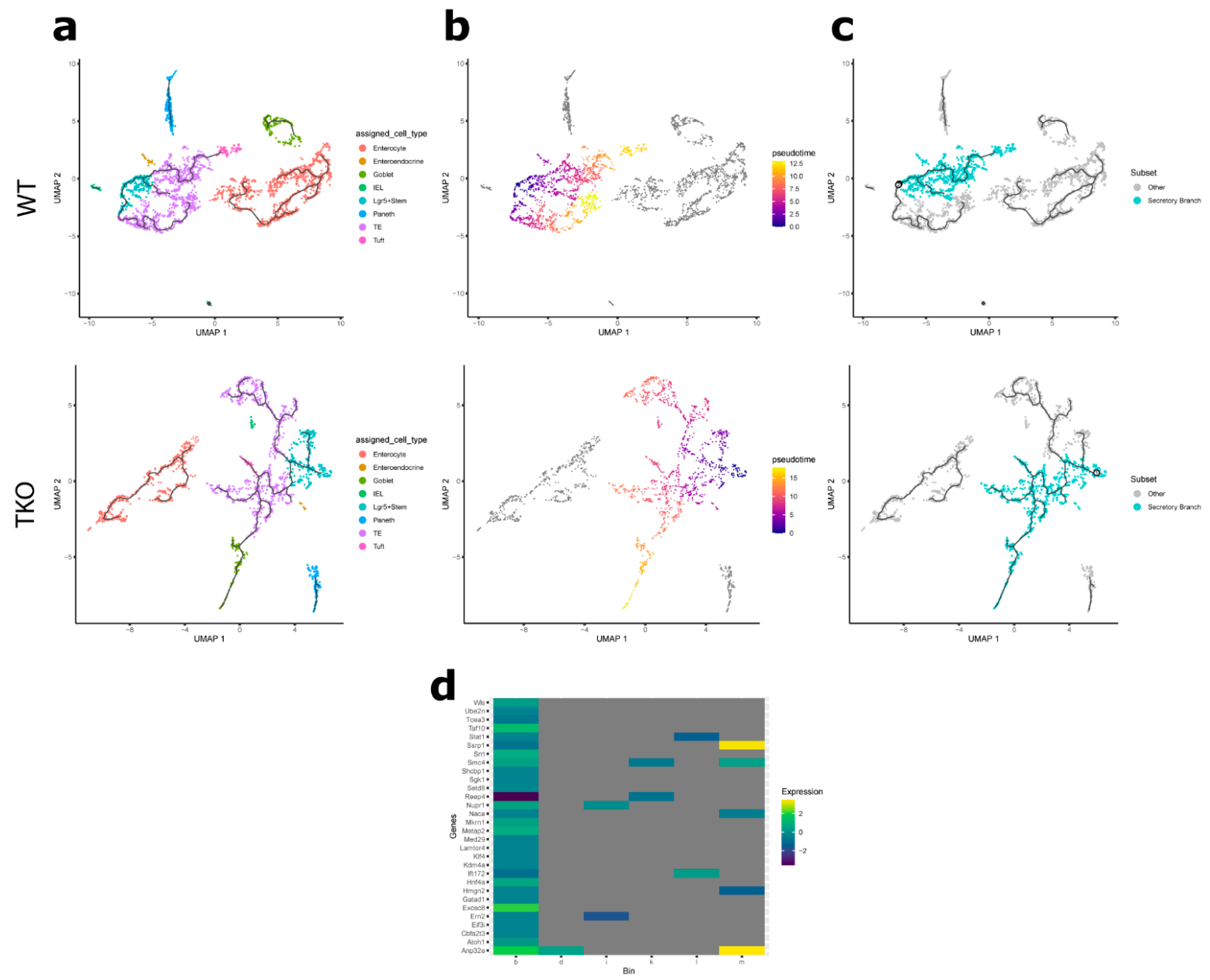

Figure 2. Identifying differentially expressed genes through pseudotime binning. (a-c) UMAP plots, black lines represent branched cell trajectories; (a) cells colored by cell type; (b) cells colored by pseudotime; (c) cells colored by selected branch for downstream analysis (blue); (d) heatmap of regression analysis slope values $\left(\beta_{0}\right)$ by genes determined to regulate expression by gene ontology analysis. $p<0.05$ for all genes shown. 


\subsection{Nupr1, Kdm4a, and Gatad1 Are Possible Novel Regulators of Secretory Cell Fate}

To investigate the kinetics of transcriptional regulators across pseudotime, the branch representing secretory precursors was chosen for further analysis based on the enriched goblet cell count in Tipe $0^{-/-}$intestine. Following subsetting, expression of each gene was fit to pseudotime across the branch in either genotype and the fits between each genotype were compared. This identified nine genes that were differentially expressed both across pseudotime within each genotype and between genotypes. Five genes (Nupr1, Klf4, Atoh1, $\mathrm{Kdm} 4 \mathrm{a}$, and Gatad1) were identified in having roles specific to differentiation (Figure 3). Plotting the polynomial fit of expression across pseudotime revealed that most of these genes demonstrated a point at which the slope of Tipe $0^{-/-}$diverged dramatically from that of the WT (Figure 3a,c). This change could represent a switch between expression programs responsible for cell fate decisions. Notably, in the $\mathrm{TIPE}^{-/-} \mathrm{UMAP}_{\text {plot, goblet }}$ cells are at the end of the secretory trajectory whereas in that of the WT, tuft cells are the end of the trajectory. Therefore, it is possible that the differential expression of these genes is due to this difference in clustering. However, this does not rule out the possible role of TIPE0. Expression of proteins known to regulate cell fate decisions demonstrate a similar fit, supporting the possible role of the identified genes in this process. For example, Spdef causes expansion of goblet cells and a reduction of Paneth cells when overexpressed in the small intestine [11]. The plot of Spdef expression vs. pseudotime demonstrates a remarkably similar shape to the plots in Figure 3a,c (data not shown). Additionally, conditional loss of Sox4 resulted in loss of tuft and enteroendocrine cells and impaired tuft cell hyperplasia after helminths infection [29]. The plot of Sox4 expression vs. pseudotime also demonstrates a similar shape to the plots of Gatad1 and Kdm4a, despite its high expression in ISCs.

Lastly, we attempted to validate the findings using RT-qPCR of previously generated small intestinal WT and Tipe $0^{-/-}$RNA samples for changes in Nupr1, Kdm4a, and Gata1 between them; however, the results were inconclusive (data not shown). We believe that the cell-specific expression of these genes causes differences in their expression to be drowned out in the analysis of pooled cells. 

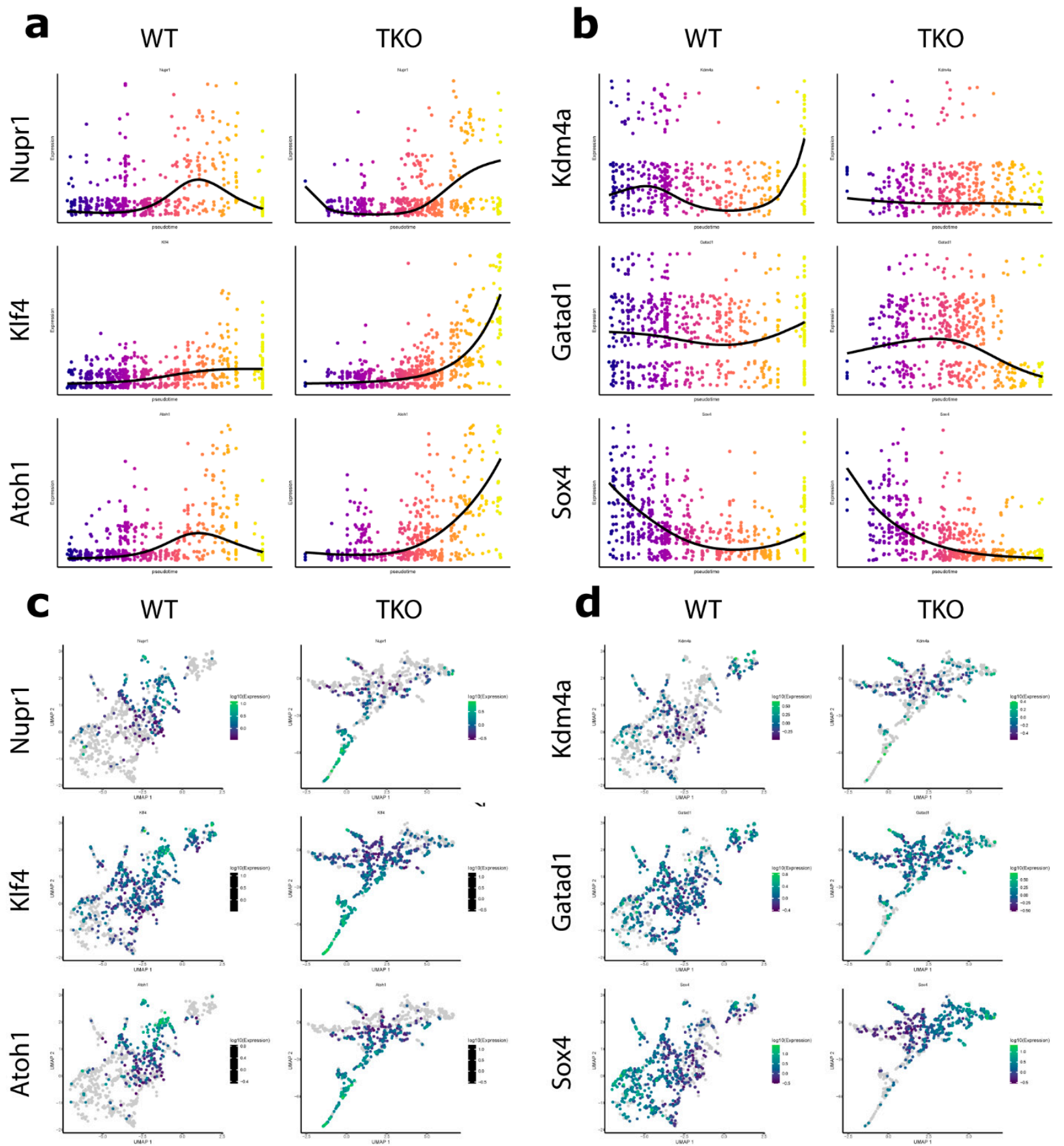

Figure 3. Genes differentially expressed across psuedotime in the secretory branch of TKO. (a,c) Plots of expression vs. pseudotime for genes upregulated (a) or downregulated (c) in TKO. Line represents the polynomial regression of expression vs. psuedotime. Cells are shown, colored by pseudotime (blue to yellow). Difference between the slope of TKO and WT fits is shown along with p-values; $(\mathbf{b}, \mathbf{d})$ UMAP of cell data set branch subsets. Cells are colored by expression of genes upregulated (b) or downregulated (d) in TKO. Cells are colored by gene expression.

\section{Discussion}

Loss of TIPE0 alters intestinal patterns of differentiation, generally shifting cells towards a more undifferentiated state. The intestinal epithelium in Tipe $0^{-1-}$ is enriched in Lgr5+ stem cells and partially differentiated cells, whereas terminally differentiated cells such as Paneth, tuft, and enteroendocrine cells are reduced. Goblet cells are the exception to this and are more abundant in the Tipe $0^{-/-}$intestinal epithelium. Interestingly, the 
clustering of WT and Tipe $0^{-/-}$cells yielded secretory trajectories that terminate in different cell types. This allowed for the investigation of regulators of cell fate decisions that produce each of these cell types as well as the role TIPE0 may play in this process.

Both Atoh1 and Klf4 have been shown to be involved in goblet cell differentiation, supporting the data found in this study. Loss of Atoh1 (also named Math1) in mice led to depletion of secretory cells (goblet, Paneth, and enteroendocrine) without affecting enterocytes. Additionally, co-localization of Atoh1 and Ki-67 in some proliferating cells suggests that secretory cells arise from a shared lineage of Atoh1-expressing precursors [3]. Intestine-specific knockout of Atoh1 yielded a similar loss of secretory cells [4]. Klf4 ${ }^{-/}$ mice demonstrated a 90\% decrease in goblet cell count while all other epithelial cell types remained constant [9]. Intestine-specific deletion of Klf4 also led to failure of goblet cell differentiation [30]. Recently, specific deletion of Klf4 in Bmi+ reserve stem cells along with parallel lineage tracing led to a reduction in goblet cells in the Bmi+ lineage, suggesting that Klf4 is able to regulate goblet cell differentiation in Bmi+ stem cell lineages [10].

Nupr1, however, is less clearly associated with goblet cell development. Nupr1 is a transcription factor responsible for mediating stress-responses. It has been found to play a role in pancreatic tumorigenesis as well as in the protection of cancer cells from stress induced death by inhibiting apoptosis [31]. Additionally, loss of Nupr1 led to decreased expression of endoplasmic reticulum stress response associated genes [32]. In the small intestine, researchers found that Nupr1 might play a role in Paneth cell differentiation and function using single-cell genomics [33]. However, the data from this study suggest that Nupr1 expression precedes differentiation to Paneth cells and is also present in Goblet cells. This may indicate that Nupr1 plays a role in directing goblet/Paneth precursor differentiation. Interestingly, Nupr1 has been found to bind to RING1B, part of the Polycomb repressive complex 1 that has been shown to promote intestinal stem cell self-renewal $[34,35]$. Despite this, the exact role Nupr1 plays in cell fate decisions remains unclear.

The distinct requirements for tuft cell differentiation have yet to be determined. Intestine-specific knockout of Atoh1 has been shown to either increase [13] or ablate [36] tuft cells in the small intestine. Inhibition of Notch signaling, which increases Atoh1 activity, was also shown to increase the number of tuft cells in the intestine [37]. This study suggests a model in which tuft cells arise from secretory precursors, in agreement with the findings of Gerbe, F. et al. [36] and VanDussen, K. et al. [37], and subsequent loss of Atoh1 expression is involved in determination of tuft cells. This is supported by the trajectory in the WT in which the immediate precursors to tuft cells are Atoh1+. Additionally, Sox4 has been identified as a possible regulator of tuft and enteroendocrine cell differentiation [27]. This study identifies two other proteins that may play a role in Tuft cell differentiation: Kdm4a and Gatad1. Kdm4a has been identified as a H3K9Me3 and H3K36Me3 specific demethylase $[38,39]$. It has already been shown to regulate cell fate decisions in skeletal muscle cell, pancreatic cell, and embryonic stem cell differentiation [40-42]. Gatad1 has been proposed to function as a histone deacetylase [43]. However, recent work has shown that it may be part of a transcriptionally repressive histone complex, regulating transcription through indirect interaction with $\mathrm{H} 3 \mathrm{~K} 4 \mathrm{Me} 3$ through $\mathrm{Kdm} 5 \mathrm{a}$ [44]. Gatad1 has also been shown to regulate mammalian lens and liver cell differentiation [45,46]. Additionally, Gatad1 was found to modulate PI3K/Akt signaling activity in hepatocellular carcinoma cells through upregulation of phosphatase of regenerating liver 3 (PRL3) by binding its promoter [47]. Both Kdm4a and Gatad1 represent possible novel regulators of tuft cell differentiation through epigenetic modulation of secretory precursors. The precise mechanism of their regulation and role in this process has yet to be determined. Additionally, it must be noted that increased baseline inflammation in the Tipe $0^{-/-}$intestine may influence changes in the stem cell niche and differences seen in terminal differentiation [22]. Further, without heterozygous littermate controls, differences in the composition of the intestinal microbiome may have influenced patterns of differentiation as well. Although the mechanism of the differential expression of Nupr1, Gatad1, and Kdm4a in the WT and Tipe0 ${ }^{-/-}$requires 
further investigation, each proposed regulator's trajectory is consistent with a role in the terminal differentiation of either goblet or tuft cells. Further work will also need to be done to validate these findings, first by sorting populations of goblet and tuft cells from WT and Tipe $0^{-/-}$ileums and then by performing downstream RT-PCR and protein analyses.

The role of TIPE0 in modulating PI3K/Akt signaling, as well as the observed changes in the cellular composition of the Tipe $0^{-/-}$intestinal epithelium, suggest that TIPE0 may play a role in the modulation of intestinal epithelial cell differentiation. The shift towards undifferentiated cells in Tipe $0^{-/}$- likely results both from increased proliferative signaling by the PI3K/Akt pathway as well as crosstalk with the Wnt/B-catenin pathway that promotes stemness. However, how this pathway modulates later cell fate decisions is currently unknown.

This study has further supported the effect of TIPE0 knockout on intestinal epithelial composition and in mediating changes in expression patterns that alter intestinal epithelial cell differentiation. This study also has identified three possible novel regulators of terminal secretory differentiation: Nupr1 in goblet cell differentiation and both Kdm4a and Gatad1 in tuft cell differentiation.

Author Contributions: Conceptualization, R.H. and J.R.G.; methodology, R.H. and J.R.G.; software, R.H. and J.R.G.; formal analysis, R.H. and J.R.G.; investigation, R.H. and J.R.G.; resources, J.R.G. and Y.H.C.; data curation, R.H. and J.R.G.; writing-original draft preparation, R.H.; writing-review and editing, R.H. and J.R.G.; visualization, R.H. and J.R.G.; supervision, J.R.G. and Y.H.C.; project administration, J.R.G. and Y.H.C.; funding acquisition, Y.H.C. and J.R.G. All authors have read and agreed to the published version of the manuscript.

Funding: This research was funded by The National Institutes of Health grants R01-AI121166, R01-AI136945, F32-DK116528, P30-DK050306, NIH-T32-CA009140.

Institutional Review Board Statement: The study was conducted according to the relevant regulatory standards and approved by the Institutional Animal Care and Use Committee of the University of Pennsylvania, protocol number 804832 on 1/29/2019.

Informed Consent Statement: No human subjects were involved in these studies.

Data Availability Statement: The data presented in this study are available from Goldsmith, J.; et al. at doi:10.1038/s41467-020-16379-2, or on request from the corresponding author.

Acknowledgments: We would like to thank Barton Poulson for training in R, and H. Sun for providing the TIPE0 KO mice. We would also like to thank Mia Levine for supporting the independent study that allowed for this research. We would also like to thank Christopher J. Lengner and Yuhua Tian for supporting the continuation of this project and Warren S. Pear for supporting the publication of this manuscript.

Conflicts of Interest: The authors declare no conflict of interest.

\section{References}

1. De Lau, W.; Barker, N.; Low, T.Y.; Koo, B.-K.; Li, V.S.W.; Teunissen, H.; Kujala, P.; Haegebarth, A.; Peters, P.J.; Van De Wetering, M.; et al. Lgr5 homologues associate with Wnt receptors and mediate R-spondin signalling. Nat. Cell Biol. 2011, 476, $293-297$. [CrossRef] [PubMed]

2. Scoville, D.H.; Sato, T.; He, X.C.; Li, L. Current View: Intestinal Stem Cells and Signaling. Gastroenterology 2008, 134, 849-864. [CrossRef] [PubMed]

3. Yang, Q.; Bermingham, N.A.; Finegold, M.J.; Zoghbi, H.Y. Requirement of Math1 for Secretory Cell Lineage Commitment in the Mouse Intestine. Science 2001, 294, 2155-2158. [CrossRef] [PubMed]

4. Shroyer, N.F.; Helmrath, M.A.; Wang, V.Y.; Antalffy, B.; Henning, S.J.; Zoghbi, H.Y. Intestine-Specific Ablation of Mouse atonal homolog 1 (Math1) Reveals a Role in Cellular Homeostasis. Gastroenterology 2007, 132, 2478-2488. [CrossRef]

5. VanDussen, K.L.; Samuelson, L.C. Mouse atonal homolog 1 directs intestinal progenitors to secretory cell rather than absorptive cell fate. Dev. Biol. 2010, 346, 215-223. [CrossRef]

6. Lee, C.S.; Perreault, N.; Brestelli, J.E.; Kaestner, K.H. Neurogenin 3 is essential for the proper specification of gastric enteroendocrine cells and the maintenance of gastric epithelial cell identity. Genes Dev. 2002, 16, 1488-1497. [CrossRef] [PubMed] 
7. Jenny, M.; Uhl, C.; Roche, C.; Duluc, I.; Guillermin, V.; Guillemot, F.; Jensen, J.; Kedinger, M.; Gradwohl, G. Neurogenin3 is differentially required for endocrine cell fate specification in the intestinal and gastric epithelium. EMBO J. 2002, 21, 6338-6347. [CrossRef]

8. López-Díaz, L.; Jain, R.N.; Keeley, T.M.; VanDussen, K.L.; Brunkan, C.S.; Gumucio, D.L.; Samuelson, L.C. Intestinal Neurogenin 3 directs differentiation of a bipotential secretory progenitor to endocrine cell rather than goblet cell fate. Dev. Biol. 2007, 309, 298-305. [CrossRef]

9. Katz, J.P.; Perreault, N.; Goldstein, B.G.; Lee, C.S.; Labosky, P.A.; Yang, V.W.; Kaestner, K.H. The zinc-finger transcription factor Klf4 is required for terminal differentiation of goblet cells in the colon. Development 2002, 129, 2619-2628.

10. Katano, T.; Bialkowska, A.B.; Yang, V.W. KLF4 Regulates Goblet Cell Differentiation in BMI1+ Reserve Intestinal Stem Cell Lineage during Homeostasis. Int. J. Stem Cells 2020, 13, 424-431. [CrossRef]

11. Noah, T.K.; Kazanjian, A.; Whitsett, J.; Shroyer, N.F. SAM pointed domain ETS factor (SPDEF) regulates terminal differentiation and maturation of intestinal goblet cells. Exp. Cell Res. 2010, 316, 452-465. [CrossRef] [PubMed]

12. Shroyer, N.F.; Wallis, D.; Venken, K.J.; Bellen, H.J.; Zoghbi, H.Y. Gfi1 functions downstream of Math1 to control intestinal secretory cell subtype allocation and differentiation. Genes Dev. 2005, 19, 2412-2417. [CrossRef] [PubMed]

13. Bjerknes, M.; Khandanpour, C.; Möröy, T.; Fujiyama, T.; Hoshino, M.; Klisch, T.J.; Ding, Q.; Gan, L.; Wang, J.; Martín, M.G.; et al. Origin of the brush cell lineage in the mouse intestinal epithelium. Dev. Biol. 2012, 362, 194-218. [CrossRef] [PubMed]

14. Spit, M.; Koo, B.-K.; Maurice, M.M. Tales from the crypt: Intestinal niche signals in tissue renewal, plasticity and cancer. Open Biol. 2018, 8, 180120. [CrossRef] [PubMed]

15. Kumar, D.; Whiteside, T.L.; Kasid, U. Identification of a Novel Tumor Necrosis Factor- $\alpha$-inducible Gene, SCC-S2, Containing the Consensus Sequence of a Death Effector Domain of Fas-associated Death Domain-like Interleukin- $1 \beta$-converting Enzymeinhibitory Protein. J. Biol. Chem. 2000, 275, 2973-2978. [CrossRef]

16. Freundt, E.C.; Bidere, N.; Lenardo, M.J. A Different TIPE of Immune Homeostasis. Cell 2008, 133, 401-402. [CrossRef]

17. Sun, H.; Gong, S.; Carmody, R.J.; Hilliard, A.; Li, L.; Sun, J.; Kong, L.; Xu, L.; Hilliard, B.; Hu, S.; et al. TIPE2, a Negative Regulator of Innate and Adaptive Immunity that Maintains Immune Homeostasis. Cell 2008, 133, 415-426. [CrossRef]

18. Zhang, X.; Wang, J.; Fan, C.; Li, H.; Sun, H.; Gong, S.; Chen, Y.H.; Shi, Y. Crystal structure of TIPE2 provides insights into immune homeostasis. Nat. Struct. Mol. Biol. 2008, 16, 89-90. [CrossRef]

19. Fayngerts, S.A.; Wu, J.; Oxley, C.L.; Liu, X.; Vourekas, A.; Cathopoulis, T.; Wang, Z.; Cui, J.; Liu, S.; Sun, H.; et al. TIPE3 Is the Transfer Protein of Lipid Second Messengers that Promote Cancer. Cancer Cell 2014, 26, 465-478. [CrossRef] [PubMed]

20. Antony, P.; Baby, B.; Vijayan, R. Molecular insights into the binding of phosphoinositides to the TH domain region of TIPE proteins. J. Mol. Model. 2016, 22, 272. [CrossRef] [PubMed]

21. Lin, M. Roles of TNFAIP in Cell Migration and Phosphoinositide Signaling. Ph.D. Thesis, University of Pennsylvania, Philadelphia, PA, USA, 2020.

22. Goldsmith, J.R.; Spitofsky, N.; Zamani, A.; Hood, R.; Boggs, A.; Li, X.; Li, M.; Reiner, E.; Ayyaz, A.; Etwebi, Z.; et al. TNFAIP8 controls murine intestinal stem cell homeostasis and regeneration by regulating microbiome-induced Akt signaling. Nat. Commun. 2020, 11, 1-20. [CrossRef] [PubMed]

23. Porturas, T.P.; Sun, H.; Buchlis, G.; Lou, Y.; Liang, X.; Cathopoulis, T.; Fayngerts, S.; Johnson, D.S.; Wang, Z.; Chen, Y.H. Crucial Roles of TNFAIP8 Protein in Regulating Apoptosis andListeriaInfection. J. Immunol. 2015, 194, 5743-5750. [CrossRef] [PubMed]

24. Trapnell, C.; Cacchiarelli, D.; Grimsby, J.; Pokharel, P.; Li, S.; Morse, A.M.; Lennon, N.J.; Livak, K.J.; Mikkelsen, T.S.; Rinn, J.L. The dynamics and regulators of cell fate decisions are revealed by pseudotemporal ordering of single cells. Nat. Biotechnol. 2014, 32, 381-386. [CrossRef] [PubMed]

25. Qiu, X.; Hill, A.; Packer, J.; Lin, D.; Ma, Y.-A.; Trapnell, C. Single-cell mRNA quantification and differential analysis with Census. Nat. Methods 2017, 14, 309-315. [CrossRef] [PubMed]

26. Qiu, X.; Mao, Q.; Tang, Y.; Wang, L.; Chawla, R.; Pliner, H.A.; Trapnell, C. Reversed graph embedding resolves complex single-cell trajectories. Nat. Methods 2017, 14, 979-982. [CrossRef] [PubMed]

27. Haber, A.L.; Biton, M.; Rogel, N.; Herbst, R.H.; Shekhar, K.; Smillie, C.; Burgin, G.; Delorey, T.M.; Howitt, M.R.; Katz, Y.; et al. A single-cell survey of the small intestinal epithelium. Nat. Cell Biol. 2017, 551, 333-339. [CrossRef] [PubMed]

28. Traag, V.A.; Waltman, L.; Van Eck, N.J. From Louvain to Leiden: Guaranteeing well-connected communities. Sci. Rep. 2019, 9, 1-12. [CrossRef]

29. Gracz, A.D.; Samsa, L.A.; Fordham, M.J.; Trotier, D.C.; Zwarycz, B.; Lo, Y.-H.; Bao, K.; Starmer, J.; Raab, J.R.; Shroyer, N.F.; et al. Sox4 Promotes Atoh1-Independent Intestinal Secretory Differentiation Toward Tuft and Enteroendocrine Fates. Gastroenterology 2018, 155, 1508-1523.e10. [CrossRef]

30. Ghaleb, A.M.; McConnell, B.B.; Kaestner, K.H.; Yang, V.W. Altered intestinal epithelial homeostasis in mice with intestine-specific deletion of the Krüppel-like factor 4 gene. Dev. Biol. 2011, 349, 310-320. [CrossRef]

31. Hamidi, T.; Algül, H.; Cano, C.E.; Sandi, M.J.; Molejon, M.I.; Riemann, M.; Calvo, E.L.; Lomberk, G.; Dagorn, J.-C.; Weih, F.; et al. Nuclear protein 1 promotes pancreatic cancer development and protects cells from stress by inhibiting apoptosis. J. Clin. Investig. 2012, 122, 2092-2103. [CrossRef]

32. Santofimia-Castaño, P.; Lan, W.; Bintz, J.; Gayet, O.; Carrier, A.; Lomberk, G.; Neira, J.L.; González, A.; Urrutia, R.; Soubeyran, P.; et al. Inactivation of NUPR1 promotes cell death by coupling ER-stress responses with necrosis. Sci. Rep. 2018, 8, 1-16. [CrossRef] 
33. Mead, B.E.; Ordovas-Montanes, J.; Braun, A.P.; Levy, L.E.; Bhargava, P.; Szucs, M.J.; Ammendolia, D.A.; MacMullan, M.A.; Yin, X.; Hughes, T.K.; et al. Harnessing single-cell genomics to improve the physiological fidelity of organoid-derived cell types. $B M C$ Biol. 2018, 16, 1-24. [CrossRef] [PubMed]

34. Santofimia-Castaño, P.; Rizzuti, B.; Pey, A.L.; Soubeyran, P.; Vidal, M.; Urrutia, R.; Iovanna, J.L.; Neira, J.L. Intrinsically disordered chromatin protein NUPR1 binds to the C-terminal region of Polycomb RING1B. Proc. Natl. Acad. Sci. USA 2017, 114, E6332-E6341. [CrossRef] [PubMed]

35. Chiacchiera, F.; Pasini, D. Control of adult intestinal identity by the Polycomb repressive machinery. Cell Cycle 2016, 16, 243-244. [CrossRef] [PubMed]

36. Gerbe, F.; Van Es, J.H.; Makrini, L.; Brulin, B.; Mellitzer, G.; Robine, S.; Romagnolo, B.; Shroyer, N.F.; Bourgaux, J.-F.; Pignodel, C.; et al. Distinct ATOH1 and Neurog3 requirements define tuft cells as a new secretory cell type in the intestinal epithelium. J. Cell Biol. 2011, 192, 767-780. [CrossRef] [PubMed]

37. VanDussen, K.L.; Carulli, A.J.; Keeley, T.M.; Patel, S.R.; Puthoff, B.J.; Magness, S.T.; Tran, I.T.; Maillard, I.; Siebel, C.; Kolterud, A.; et al. Notch signaling modulates proliferation and differentiation of intestinal crypt base columnar stem cells. Development 2011, 139, 488-497. [CrossRef]

38. Bavetsias, V.; Lanigan, R.M.; Ruda, G.F.; Atrash, B.; McLaughlin, M.G.; Tumber, A.; Mok, N.Y.; Le Bihan, Y.-V.; Dempster, S.; Boxall, K.J.; et al. 8-Substituted Pyrido[3,4-d]pyrimidin-4(3H)-one Derivatives As Potent, Cell Permeable, KDM4 (JMJD2) and KDM5 (JARID1) Histone Lysine Demethylase Inhibitors. J. Med. Chem. 2016, 59, 1388-1409. [CrossRef]

39. Cardamone, M.D.; Tanasa, B.; Chan, M.; Cederquist, C.T.; Andricovich, J.; Rosenfeld, M.G.; Perissi, V. GPS2/KDM4A Pioneering Activity Regulates Promoter-Specific Recruitment of PPAR $\gamma$. Cell Rep. 2014, 8, 163-176. [CrossRef] [PubMed]

40. Verrier, L.; Escaffit, F.; Chailleux, C.; Trouche, D.; Vandromme, M. A New Isoform of the Histone Demethylase JMJD2A/KDM4A Is Required for Skeletal Muscle Differentiation. PLoS Genet. 2011, 7, e1001390. [CrossRef]

41. Liu, J.; Banerjee, A.; Herring, C.A.; Attalla, J.; Hu, R.; Xu, Y.; Shao, Q.; Simmons, A.J.; Dadi, P.K.; Wang, S.; et al. Neurog3Independent Methylation Is the Earliest Detectable Mark Distinguishing Pancreatic Progenitor Identity. Dev. Cell 2019, 48, 49-63.e7. [CrossRef]

42. Wu, L.; Wary, K.K.; Revskoy, S.; Gao, X.; Tsang, K.; Komarova, Y.A.; Rehman, J.; Malik, A.B. Histone Demethylases KDM4A and KDM4C Regulate Differentiation of Embryonic Stem Cells to Endothelial Cells. Stem Cell Rep. 2015, 5, 10-21. [CrossRef]

43. Theis, J.L.; Sharpe, K.M.; Matsumoto, M.E.; Chai, H.S.; Nair, A.A.; Theis, J.D.; De Andrade, M.; Wieben, E.D.; Michels, V.V.; Olson, T.M. Homozygosity Mapping and Exome Sequencing Reveal GATAD1 Mutation in Autosomal Recessive Dilated Cardiomyopathy. Circ. Cardiovasc. Genet. 2011, 4, 585-594. [CrossRef] [PubMed]

44. Knight, H.C. Alternative Non-canonical Translation Initiation Codons are Used to Synthesize Novel Isoforms. Ph.D. Thesis, University of Southampton, Southampton, UK, April 2017.

45. Zhao, Y.; Zheng, D.; Cvekl, A. Profiling of chromatin accessibility and identification of general cis-regulatory mechanisms that control two ocular lens differentiation pathways. Epigenetics Chromatin 2019, 12, 27. [CrossRef] [PubMed]

46. Su, X.; Shi, Y.; Zou, X.; Lu, Z.-N.; Xie, G.; Yang, J.Y.H.; Wu, C.-C.; Cui, X.-F.; He, K.-Y.; Luo, Q.; et al. Single-cell RNA-Seq analysis reveals dynamic trajectories during mouse liver development. BMC Genom. 2017, 18, 1-14. [CrossRef] [PubMed]

47. Sun, W.; Zhang, Y.; Wong, K.C.; Liu, K.; Yang, Y.; Wu, B.; Tong, J.H.; Chan, A.W.; Chan, H.L.; Yu, J. Increased expression of GATA zinc finger domain containing 1 through gene amplification promotes liver cancer by directly inducing phosphatase of regenerating liver 3. Hepatology 2017, 67, 2302-2319. [CrossRef] 